\title{
A Curvature Compensation Technique for Bandgap Voltage References Using Adaptive Reference Temperature
}

\author{
Kee-Chee Tiew, Jim Cusey*, Randall Geiger \\ Department of Electrical and Computer Engineering, IOWA STATE UNIVERSITY, Ames, IOWA 50011, USA \\ ${ }^{*}$ Dallas Semiconductor, Dallas, TEXAS, USA
}

\begin{abstract}
A curvature compensation technique for bandgap voltage references that utilizes an adaptive reference temperature is presented. This compensation technique is intended for high-resolution temperature-stable analogdigital converters. A test circuit had been designed in a $0.6 \mu \mathrm{m}$ CMOS technology to implement this technique. The reference voltage has a simulated temperature coefficient of $2.3 \mathrm{ppm} /{ }^{\circ} \mathrm{C}$ over the temperature range of -40 to 90 ${ }^{\circ} \mathrm{C}$.
\end{abstract}

\section{INTRODUCTION}

Voltage reference circuits are widely used 1 in data converters. Bandgap voltage references (BVR) have become the most popular solution since they were first introduced in the 80's. However, even the BVR have performance limitations due to the nonlinear temperature variation of its output voltage. The temperature variation of the BVR output voltage is illustrated in Fig. 1 where the deviation in output voltage due to the "curvature" over the temperature range from $20 \mathrm{C}$ to $100 \mathrm{C}$ is typically around $35 \mathrm{ppm}$ even for a basic well-designed reference. This deviation is too large for many applications. Several approaches [3], [5], [6] have been proposed to correct the curvature of the BVR output voltage $\left(\mathrm{V}_{\mathrm{REF}}\right)$. The proposed solutions attempt to cancel the nonlinear components of $\mathrm{V}_{\mathrm{REF}}$ using continuous and differentiable higher-order temperature terms. These correction terms are either difficult to generate or unsuitable for standard CMOS process [3],[6]. Moreover, these solution are sensitive to process variations because of the need to closely match the nonlinear coefficients of opposite signs in order to have decent cancellation [5],[6]. In this paper, a curvature compensation alternative that does not utilize continuous and differential nonlinear cancellation is presented.

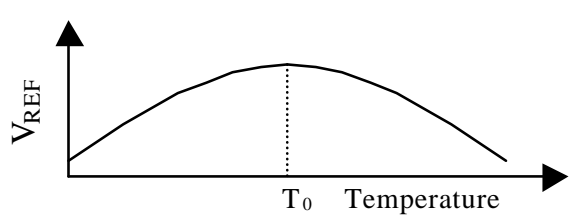

Fig. 1 Temperature variation of BVR output voltage

\section{BANDGAP VOLTAGE REFERENCE}

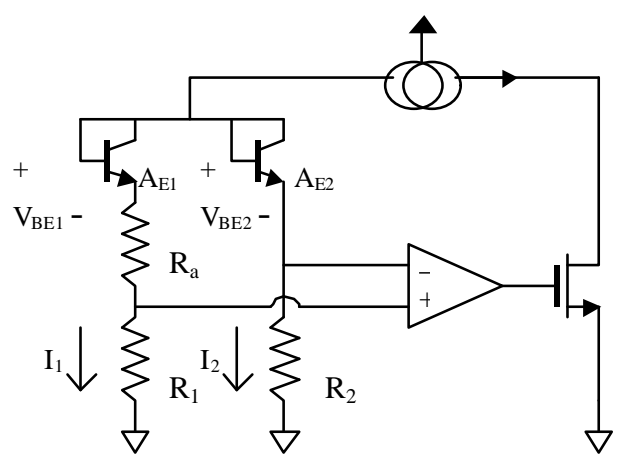

Fig. 2 Two diodes that generate $\mathrm{V}_{\mathrm{BE}}$ and PTAT voltage

Fig. 2 shows a standard BVR circuit with two diode-connected BJTs, which have a baseemitter area ratio of $A_{E 1} / A_{E 2}$. The closed-loop op amp forces the same voltage drop across $R_{1}$ and $R_{2}$. The temperature characteristic of $V_{B E}$ is governed by the following expression [2],[4]:

$$
\begin{aligned}
& V_{B E}(T)=V_{G_{0}}\left(1-\frac{T}{T_{0}}\right)+\left(\frac{T}{T_{0}}\right) V_{B E_{0}} \\
& -\eta\left(\frac{k T}{q}\right) \ln \left(\frac{T}{T_{0}}\right)+\left(\frac{k T}{q}\right) \ln \left(\frac{J_{C}}{J_{C_{0}}}\right)
\end{aligned}
$$

where $\mathrm{V}_{\mathrm{G} 0}$ is the silicon bandgap voltage extrapolated to $0{ }^{\circ} \mathrm{K}(\sim 1.206 \mathrm{~V}), \mathrm{k}$ is the Boltzmann's constant, $\eta$ is a process dependent constant, $\mathrm{T}_{0}$ denotes the reference 
temperature, $\mathrm{V}_{\mathrm{BEO}}$ is the base-emitter voltage drop at the reference temperature and $\mathrm{J}_{\mathrm{C} 0}$ is the collector current density evaluated at the reference temperature $\mathrm{T}_{0}$. A PTAT voltage (proportional to absolute temperature) can be derived from the difference between the $V_{B E}$ drops of two transistors. If follows from (1) that:

$$
V_{P T A T}=\Delta V_{B E}=V_{B E 2}-V_{B E} 1
$$

where

$$
\Delta V_{B E}=\frac{k T}{q} \ln \left(\frac{A_{E_{1}}}{A_{E_{2}}} \cdot \frac{I_{2}}{I_{1}}\right)
$$

In (3), the collector current density $\mathbf{J}_{\mathrm{C}}$ has been replaced by $J_{C}=I / A_{E}$ where $A_{E}$ is the effective base-emitter area. $V_{R E F}$ is then obtained by adding the PTAT voltage $\left(\Delta \mathrm{V}_{\mathrm{BE}}\right)$ to the "inversely" PTAT voltage $\left(\mathrm{V}_{\mathrm{BE}}\right)$, which yields the following expression:

$$
V_{R E F}=V_{B E}+K \cdot \Delta V_{B E}
$$

$\mathrm{K}$ is chosen to cancel the first order temperature variation of $\mathrm{V}_{\mathrm{BE}}$. In the implementation of this paper, a switchedcapacitor circuit as depicted in Fig. 3 was used to perform the weighted addition of (4).

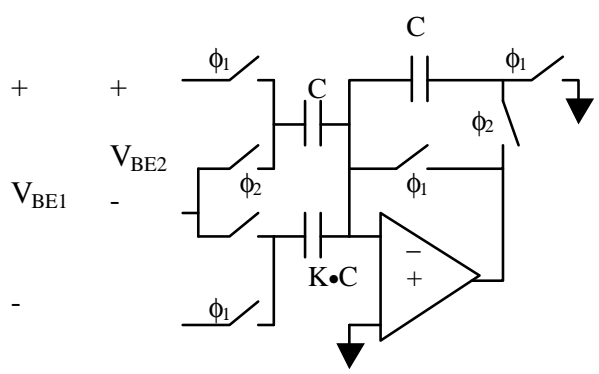

Fig. 4 Switched-capacitor circuit that performs weighted addition

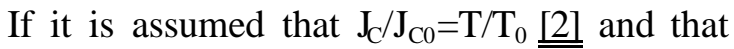
value of $\mathrm{K}$ is chosen to cancel the first-order temperature, (4) can be expanded and rewritten as [2]:

$$
V_{R E F}=V_{G 0}+(\eta-1) \frac{k T}{q}\left[1+\ln \left(\frac{T_{0}}{T}\right)\right]
$$

The second term in (5) is the nonlinear component of $\mathrm{V}_{\mathrm{REF}}$. To examine the temperature variation of $\mathrm{V}_{\mathrm{REF}}$, the expression for $\partial \mathrm{V}_{\mathrm{REF}} / \partial \mathrm{T}$ is derived [2]:

$$
\frac{\partial V_{R E F}}{\partial T}=(\eta-1) \frac{k}{q} \ln \left(\frac{T_{0}}{T}\right)
$$

As (6) describes, $V_{\text {REF }}$ has zero temperature variation $\left(\partial \mathrm{V}_{\mathrm{REF}} / \partial \mathrm{T}=0\right)$ at $\mathrm{T}=\mathrm{T}_{0}$ and $\mathrm{V}_{\mathrm{REF}}$ has minimal variation with temperature for $T$ around $\mathrm{T}_{0}$. Therefore, if a BVR circuit has an adaptive $T_{0}$ that varies over temperature, then a $V_{R E F}$ with a minimal temperature variation over a wide temperature range can be realizable.

\section{CURVATURE COMPENSATION}

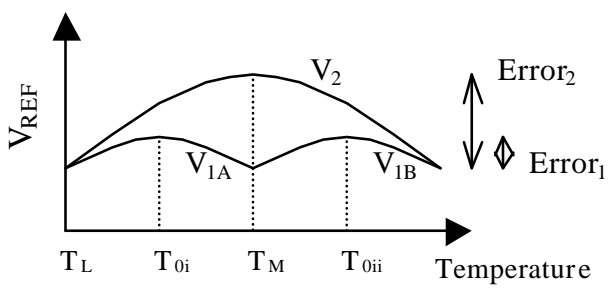

Fig. 4 BVR circuit with adaptive $\mathrm{T}_{0}$

The assumed relationship of $\mathrm{J}_{\mathrm{C}} / \mathrm{J}_{\mathrm{C} 0}=\mathrm{T} / \mathrm{T}_{0}$ [2] can be readily realized at the circuit level. This relationship suggests that $T_{0}$ is adjustable through changing the DC bias current of the BVR circuit. Assume that the circuit in Fig. 2 is designed to operate with a reference temperature at $T_{0 \mathrm{i}}$ when the temperature range is between $T_{L}$ and $T_{M}$ as illustrated in Fig. 4 . When the temperature range gets above $T_{M}$, the BVR circuit will be calibrated such that it will now operate with a reference temperature at $\mathrm{T}_{0 \mathrm{ii}}$. Overall, the BVR with adaptive $\mathrm{T}_{0}$ has a two-segment $\mathrm{V}_{\mathrm{REF}}$ curve designated by $\mathrm{V}_{1 \mathrm{~A}}$ and $\mathrm{V}_{1 \mathrm{~B}}$ in Fig. 4. With the segmented correction, the maximum error caused by the curvature is Error ${ }_{1}$. This can be contrasted to the conventional BVR that is referenced to temperature $T_{M}$ and has the $V_{\text {REF }}$ curve of $V_{2}$ as depicted in Fig. 4 with a maximum error of Error $_{2}$. This illustration demonstrates that the adaptive $\mathrm{T}_{0}$ BVR compensation technique significantly mproves the $V_{R E F}$ accuracy over 
a wide temperature range. To further improve the $\mathrm{V}_{\mathrm{REF}}$ accuracy, the overall temperature range can be partitioned into additional regions each of which spans a correspondingly smaller temperature range. In this work, the temperature range was partitioned into four regions (quadrants). To address these four regions, an ADC needs to perform a two-bit temperature-digital signal conversion. The region selection has been implemented in a two-step process. In the first step, an uncalibrated BVR is used to generate the reference voltage of the ADC that is used to sense temperature. The digital signal is then fed to the BVR to select the proper quadrant as interpreted by the 2 bit digital signal. With the BVR compensated, the ADC is now ready for doing data conversion. The system block diagram is shown in Fig. 5.

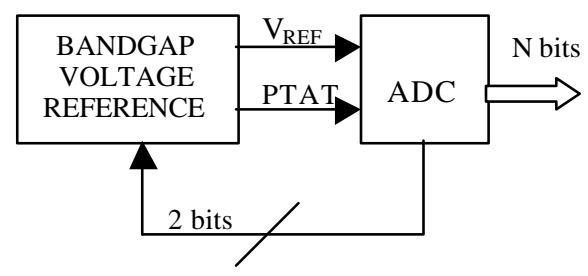

Fig. 5 System block diagram for BVR curvature compensation

\section{CIRCUIT IMPLEMENTATION}

Consider the circuit in Fig. 6, which is a slight modification from the circuit in Fig. 2. The switches $S_{0}$ to $S_{3}$ are controlled by the 2 bits digital signal that assigns the appropriate temperature quadrant. The resistances $\mathrm{R}_{10}, \mathrm{R}_{11}$, $R_{12}$ and $R_{13}$ are sized such that $\mathrm{R}_{10}<\mathrm{R}_{11}<\mathrm{R}_{12}<\mathrm{R}_{13}$. Calibration is implemented by selecting the appropriate resistor to adjust the current in the left-most transistor of Fig. 6. As indicated by the relationship $\mathrm{J}_{\mathrm{C}} / \mathrm{J}_{\mathrm{C} 0}=\mathrm{T} / \mathrm{T}_{0}$, adjusting $\mathrm{J}_{\mathrm{C}}$ results in a change of $\mathrm{T}_{0}$ is mentioned. Although adjusting $\mathrm{J}_{\mathrm{C}}$ accomplishes the desired goal of moving $\mathrm{T}_{0}$ along the horizontal axis, it can be shown that the magnitude of the peak value of $\mathrm{V}_{\mathrm{REF}}$ in any quadrant is also affected by $\mathrm{J}_{\mathrm{C}}$. If the temperature is in the first quadrant, then $S_{0}$ is closed and the circuit has a reference temperature at $\mathrm{T}_{01}$ as depicted by the segment labeled $\mathrm{V}_{\mathrm{REF} 1}$ in Fig. 7a. Likewise, if the temperature is in the second quadrant, $\mathrm{S}$ is open and $S_{1}$ is closed to increase the effective resistance $R_{1}$. This action has the net effect of increasing the current flowing through the BJT pair. By doing so, the reference temperature of the BVR circuit is shifted from $T_{01}$ to $T_{02}$ and so on. In order to align the peak values of the four $V_{R E F}$ curves of different $T_{0}$ to realize a flat $\mathrm{V}_{\mathrm{REF}}$, $\mathrm{DC}$ level adjustment is required, as indicated in Fig. 7b. This is realized through sizing the feedback capacitor dynamically in the switched-capacitor circuit depicted in Fig. 3.

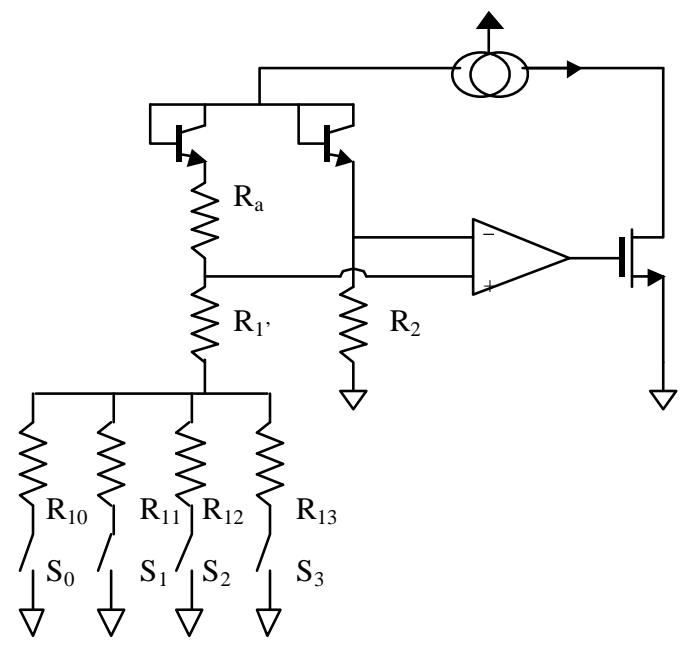

Fig. 6 Modified BVR circuit that implements adaptive $T_{0}$ compensation technique

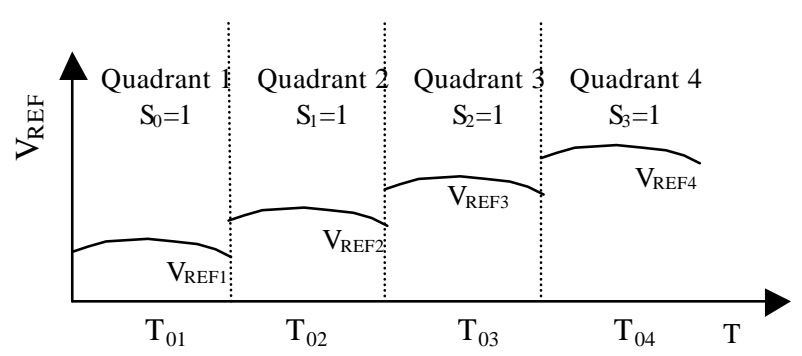

Fig. 7a $V_{\text {REF }}$ measured from the circuit in Fig. 5

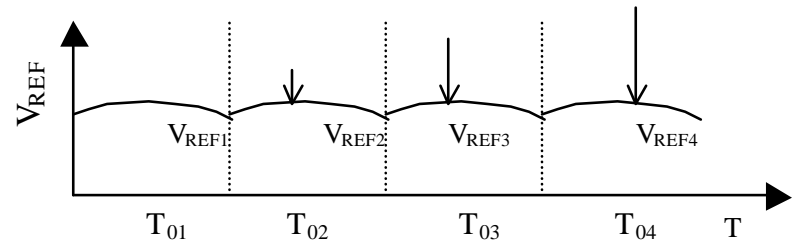

Fig. 7b Flat $V_{\text {REF }}$ is realized after DC level adjustment 
The circuit has been designed, laid-out and is currently awaiting fabrication. The output of the voltage reference, $\mathrm{V}_{\mathrm{REF}}$, has been simulated in HSPICE. Simulation results of the circuit extracted from the layout predict a temperature coefficient of at most $2.3 \mathrm{ppm} /{ }^{\circ} \mathrm{C}$ over the temperature range of -40 to $90{ }^{\circ} \mathrm{C}$. The simulated $\mathrm{V}_{\mathrm{REF}}$ varies between $1.2166 \mathrm{~V}$ to $1.2169 \mathrm{~V}$. The chip occupies an area of 0.39 $\mathrm{mm}^{2}$, and consumes $0.756 \mathrm{~mW}$ at $90{ }^{\circ} \mathrm{C}$ and $0.468 \mathrm{~mW}$ at $-40{ }^{\circ} \mathrm{C}$ with a single supply voltage of $\mathrm{V}_{\mathrm{DD}}=3 \mathrm{~V}$.

\section{DISCUSSION}

The proposed BVR circuit is affected by other non-idealities, specifically the offset voltage of the op amp and process parameter variations. The offset voltage causes the voltage drops across $R_{1}$ and $R_{2}$ to be different. The error caused by the offset voltage of the op amp has been compensated with a chopper technique [1]. Process variations such as sheet resistance deviation and process corner differences can move the reference temperature in each temperature quadrant away from the quadrant center causing the curvature error to increase. To counteract this affect, $R_{a}$ and $R_{2}$ are trimmed using switches similar to those depicted in Fig. 6 to center the reference temperature in each temperature quadrant.

\section{CONCLUSION}

A BVR curvature compensation scheme using an adaptive reference temperature circuit has been presented. This technique is easy to implement and trim in contrast to conventional continuous and differentiable curvature compensation techniques.

\section{REFERENCES}

[1] A. Bakker, J. Huijsing, High-Accuracy CMOS Smart Temperature Sensors, Kluwer Academic, 2000.

[2] D.A. Johns, K. Martin, Analog Integrated Circuit Design, John Wiley \& Sons, 1997.
[3] M. Gu nawan, G.C.M. Meijer, J. Fonderie, and J.H. Huijsing, "A curvature-corrected low-voltage bandgap reference," IEEE Journal of Solid State Circuits, vol.28, no.6, pp.667-670, June 1993.

[4] Y.P. Tsividis, "Accurate analysis of temperature effects in IC-VBE characteristics with application to bandgap reference sources." IEEE Journal of Solid-State Circuits, vol.sc-15, no.6, pp. 1076-1084, Dec. 1980.

[5] M.A.P. Pertijs, A. Bakker, J.H. Huijsing, "A highaccuracy temperature sensor with second-order curvature correction and digital bus interface." ISCAS 2001, vol.1, pp. $368-371,2001$

[6] I.M. Filanovsky, Y.F. Chan, "BICMOS cascaded bandgap voltage reference," IEEE 39th Midwest symposium on Circuits and Systems, vol.2, pp. $943-946,1996$ 